\title{
Use of Chromogenic Assay of Factor X to Accept or Reject INR Results in Warfarin Treated Patients
}

\author{
Michael J. Sanfelippo, MS, MT(ASCP); Wendy Zinsmaster, RN; \\ Doris L. Scherr, BS, MT(ASCT); and Gene R. Shaw, MD
}

\begin{abstract}
A warfarin treated patient unexpectedly presented with an elevated international normalized ratio (INR). Repeat testing in two laboratories gave conflicting results. The chromogenic assay of factor $X$ was used to determine the correct INR result. The patient had laboratory results consistent with a dysfibrinogenemia, which prevented detection of the endpoint with a photooptical detection system. The chromogenic assay of factor $X$ is recommended for monitoring patients on warfarin when the INR cannot be accurately determined due to interference with the fibrin endpoint in the INR.
\end{abstract}

Keywords: Factor X; International normalized ratio (INR);Warfarin

Reprint Requests

Michael J. Sanfelippo, MS, MT(ASCP)

Marshfield Labs

1000 North Oak Avenue

Marshfield,WI 54449

Tel: 7I5-221-6320

Fax: 715-221-6339

Email: sanfelippo.michael@marshfieldclinic.org

Received: December 19, 2008

Revised: February 12, 2009

Accepted: February 18, 2009

doi: $10.3121 / \mathrm{cmr} .2009 .836$
$\mathrm{T}$ he assay of factor $\mathrm{X}$ with a chromogenic substrate has proved useful in monitoring warfarin therapy in patients with the lupus anticoagulant and patients being transitioned from direct thrombin inhibitors to warfarin. We now report the application of this test in monitoring a patient whose prothrombin time endpoint could not be detected leading to a falsely elevated international normalized ratio (INR).

\section{Methods}

Patient History

The patient was a 79-year-old female with a history of congestive heart failure and diminished lung capacity due to lung resection for lung cancer. She had an aortic valve replacement and atrial fibrillation and had been on warfarin for approximately 7 months with INRs in the therapeutic range and no complications. An unexpected pattern of INRs over 11.0 caused the anticoagulation clinic to do a reassessment of the patient's medications, general health, diet and physical activity. When this assessment failed to support the elevated INRs, the accuracy of the INRs was questioned, and the patient was called back and tested by another lab. A lack of agreement between the elevated INRs and the INR done at the second lab caused the anticoagulation clinic to consult the special coagulation laboratory.

\section{Laboratory Methods}

The prothrombin time was performed by standard methodology on a Sysmex 1500 with Dade Innovin reagent (Sysmex Corporation, Kobe, Japan) at Lab A and Lab C. Lab B used Instrumentation Laboratory Recomboplastin (ISI) and 
Table 1. INR results on patient with dysfibrinogenemia

\begin{tabular}{|c|c|c|c|}
\hline Date & INR & Lab & Factor X \\
\hline $\begin{array}{l}\text { 02/01/08, } \\
\text { 05/27/08 }\end{array}$ & $1.2-2.4$ & C & Not required \\
\hline $\begin{array}{l}\text { 08/22/08, } \\
\text { 08/26/08 }\end{array}$ & $2.9-2.4$ & A & Not required \\
\hline 09/02/08 & 11.0 & B & Not performed \\
\hline 09/03/08 & 2.2 & $A$ & Not performed \\
\hline 09/04/08 & 1.3 & A & Not performed \\
\hline 09/05/08 & 1.6 & $A$ & Not performed \\
\hline 09/06/08 & 2.4 & A & Not performed \\
\hline 09/08/08 & 11.0 & B & Not performed \\
\hline 09/09/08 & 11.0 & $B$ & Not performed \\
\hline 09/10/08 & 1.6 & B & Not performed \\
\hline 09/13/08 & 11.0 & B & Not performed \\
\hline 09/15/08 & 11.0 & B & Not performed \\
\hline 09/16/08 & 1.2 & $\mathrm{C}$ & Not performed \\
\hline 09/19/08 & 1.4 & B & Not performed \\
\hline 09/22/08 & 1.4 & B & Not performed \\
\hline 09/26/08 & 1.4 & $\mathrm{~B}$ & Not performed \\
\hline 09/30/08 & 11.0 & B & Not performed \\
\hline 10/06/08 & 11.0 & B & Not performed \\
\hline 10/06/08 & 1.3 & $\mathrm{C}$ & $79 \%$ \\
\hline 10/13/08 & 1.8 & C & Not performed \\
\hline $10 / 20 / 08$ & 2.3 & $\mathrm{C}$ & Not performed \\
\hline 10/27/08 & 2.7 & $\mathrm{C}$ & $43 \%$ \\
\hline $11 / 10 / 08$ & 2.4 & $\mathrm{C}$ & $46 \%$ \\
\hline
\end{tabular}

INR, international normalized ratio.

an ACL model 7000. The thrombin time and reptilase time were performed by standard methods on a Dade Model BCS instrument. Fibrinogen was performed by the Claus method on a Sysmex 1500.

Fibrinogen antigen was performed by radial diffusion using prepared plates purchased from The Binding Site (San Diego, $\mathrm{CA}$ ). The chromogenic assay of factor $\mathrm{X}$ was performed as previously described with the exception that it was performed on a Dade BCS. ${ }^{1}$

\section{Results}

The patient's INR results are summarized in table 1. Laboratory studies included a thrombin time of $21.4 \mathrm{sec}$ (normal 16.4$20.7 \mathrm{sec}$ ), reptilase time $16.1 \mathrm{sec}$ (normal 15.5-19.0 sec), fibrinogen activity $642 \mathrm{mg} / \mathrm{dL}$ (normal $174-442 \mathrm{mg} / \mathrm{dL}$ ) and fibrinogen antigen $750 \mathrm{mg} / \mathrm{dL}$ (normal 180-310 mg/dL).

\section{Discussion}

Warfarin sodium is one of the most widely prescribed drugs. It is also one of the most difficult to control due to interactions with other drugs and interference from an array of physiological factors. For these reasons it is monitored with the one-stage prothrombin time reported as the INR. The use of this reporting system has significantly reduced confusion regarding appropriate levels of anticoagulation and it has brought uniformity to patient monitoring when multiple laboratories are involved.

While the INR is the accepted method of monitoring warfarin therapy, there are conditions in which the results from this test can be misleading. Patients with lupus anticoagulants ${ }^{1}$ and patients being converted from anticoagulation with the direct thrombin inhibitors may have elevated INRs, making the warfarin effect difficult to access. In these settings, the assay of factor $\mathrm{X}$ with a chromogenic substrate has been used with success to monitor warfarin anticoagulation. ${ }^{2}$ We have now utilized this assay in a situation where conflicting INRs were obtained on the same patient. One laboratory sporadically reported INRs of over 11, while other laboratories reported INRs from 1.2 to 2.9 . This disparity of results led the anticoagulation clinic to refer this patient to the special coagulation laboratory for study to determine the correct INR.

A review of the patient's records showed that all the INRs of over 11 were reported from Lab B. In all, Lab B performed 11 INRs and 7 of those were over 11. The picture was obscured by an occasional INR from Lab B that was in a therapeutic or nearly therapeutic range. The anticoagulation clinic split a specimen on 10/06/08, sending one aliquot to Lab B and another to Lab C. The results, as seen in table 1, were 11.0 and 1.3 for Labs B and C, respectively. To decide the correct value, Lab $\mathrm{C}$ sent the specimen to the special coagulation laboratory.

The immediate issue as to which was the correct value was addressed by performing a factor $\mathrm{X}$ assay with a chromogenic substrate. A value of $79 \%$ was obtained which was consistent with the INR of 1.3 that had been reported by Lab C. If the INR of 11 was the correct result, the factor $\mathrm{X}$ would have been $<10 \%$. The factor $\mathrm{X}$ range which corresponds to the INR range of 2 to 3.5 is $43 \%$ to $17 \%{ }^{1}$.

Additional studies suggested that the patient may have an abnormal form of fibrinogen, or dysfibrinogenemia. This was suggested by a slightly prolonged thrombin time and an antigenic fibrinogen of approximately $100 \mathrm{mg} / \mathrm{dL}$ higher than the fibrinogen by functional assay. Some forms of dysfibrinogenemia may be associated with bleeding tendencies and other forms may have thrombotic tendencies. However, most dysfibrinogenemias are asymptomatic as was our patient. ${ }^{3}$

Difficulty in detection of a clot by certain instruments has been reported with some forms of dysfibrinogenemia. This appears to be a problem with instruments that rely solely on a photo-optical endpoint. Fibrinogen Longmont is a dysfibrinogenemia that is a cause of missed fibrin endpoints in clotting test performed on instruments with photo-optical 
detection systems. ${ }^{4}$ The patient's plasma in our case has striking similarities to Fibrinogen Longmont. The instrument used at lab B that consistently gave INRs over 11 used a photo-optical detection system. A photo-optical detection system was also used on the Sysmex 1500 used at labs A and C. The difference in ability to detect the fibrin end point between the two different makes of instruments may be in the mathematical model used to determine the true fibrin end point. The fibrin end point is determined from the measurement of the rate of change in light scatter. Each manufacturer has its own mathematical model which works optimally with its system. Some models may be less that ideal for detection of the fibrin end point when there is a defect in either fibrinopeptide release or fibrin monomer polymerization as occurs in the dysfibinogenemias.

Laboratory testing for fibrinogen, thrombin time and reptilase time on our patient's plasma was performed on the Sysmex 1500 which is capable of detecting the fibrin end point of these tests, explaining why the thrombin time was minimally prolonged, and the fibrinogen and reptilase times were within references ranges. A similar pattern of test results was reported with Fibrinogen Longmont when the patient's plasma was tested on an instrument which was capable of detecting the fibrin end point. ${ }^{4}$ The fibrinogen, thrombin time and reptilase time were all within reference ranges on the mechanical end-point instrument; however, when the thrombin time and reptilase time were performed on the ACL 3000 both tests were significantly prolonged.

Fortunately, dysfibrinogenemias are not very common. However, they will be encountered and they may cause erroneously elevated INRs in warfarin-treated patients. Under such circumstances the chromogenic assay of factor $\mathrm{X}$ is an effective means of monitoring patients and of determining the validity of an INR result.

\section{Acknowledgments}

The authors thank Marshfield Clinic Research Foundation for its support through the assistance of Marie Fleisner and Alice Stargardt in the preparation of this article.

\section{References}

1. Sanfelippo MJ, Sennet J, McMahon EJ. Falsely elevated INRs in warfarin-treated patients with the lupus anticoagulant. WMJ 2000;99:62-64, 43

2. Moll S, Ortel TL. Monitoring warfarin therapy in patients with lupus anticoagulants. Ann Intern Med 1997;127:177-185.

3. Hayes T. Dysfibrinogenemia and thrombosis. Arch Pathol Lab Med 2002;126:1387-1390.

4. Lefkowitz JB, DeBoom T, Weller A, Clarke S, Lavrinets D. Fibrinogen Longmont: a dysfibrinogenemia that causes prolonged clot-based test results only when using an optical detection method. Am J Hematol 2000;63:149-155.
Author Affiliations

Michael J. Sanfelippo, MS, MT(ASCP)

Technical Director of Coagulation Services

Marshfield Labs, Marshfield Clinic

1000 North Oak Avenue

Marshfield, Wisconsin 54449

Wendy Zinsmaster, $R N$

Assistant Manager, Anticoagulation Clinic

Marshfield Clinic Indianhead Center

1020 Lakeshore Drive

Rice Lake, Wisconsin 54868

Doris Scheer, BS, MT(ASCT)

Hematology Technical Manager

Marshfield Labs, Marshfield Clinic

1000 North Oak Avenue

Marshfield, Wisconsin 54449

Gene R. Shaw, MD

Medical Director

Marshfield Labs, Marshfield Clinic

1000 North Oak Avenue

Marshfield, Wisconsin 54449 